\title{
Measurement of pharyngeal sensory cortical processing: technique and physiologic implications
}

\author{
Inga K Teismann*1,2, Olaf Steinstraeter ${ }^{1}$, Tobias Warnecke ${ }^{2}$, E \\ Bernd Ringelstein ${ }^{2}$, Christo Pantev ${ }^{1}$ and Rainer Dziewas ${ }^{2}$
}

\author{
Address: ${ }^{1}$ Institute for Biomagnetism and Biosignalanalysis, University of Muenster, Malmedyweg 15, 48149 Muenster, Germany and \\ ${ }^{2}$ Department of Neurology, University of Muenster, Albert-Schweitzer-Str.33, 48149 Muenster, Germany \\ Email: Inga K Teismann* - i.teismann@uni-muenster.de; Olaf Steinstraeter - steinol@uni-muenster.de; \\ Tobias Warnecke - tobias.warnecke@ukmuenster.de; E Bernd Ringelstein - neurologie@uni-muenster.de; Christo Pantev - pantev@uni- \\ muenster.de; Rainer Dziewas - dziewas@uni-muenster.de \\ * Corresponding author
}

Published: 14 July 2009

BMC Neuroscience 2009, 10:76 doi:10.1/86/147|-2202-10-76
Received: 10 September 2008

Accepted: 14 July 2009

This article is available from: http://www.biomedcentral.com/147I-2202/10/76

(C) 2009 Teismann et al; licensee BioMed Central Ltd.

This is an Open Access article distributed under the terms of the Creative Commons Attribution License (http://creativecommons.org/licenses/by/2.0), which permits unrestricted use, distribution, and reproduction in any medium, provided the original work is properly cited.

\begin{abstract}
Background: Dysphagia is a major complication of different diseases affecting both the central and peripheral nervous system. Pharyngeal sensory impairment is one of the main features of neurogenic dysphagia. Therefore an objective technique to examine the cortical processing of pharyngeal sensory input would be a helpful diagnostic tool in this context. We developed a simple paradigm to perform pneumatic stimulation to both sides of the pharyngeal wall. Whole-head MEG was employed to study changes in cortical activation during this pharyngeal stimulation in nine healthy subjects. Data were analyzed by means of synthetic aperture magnetometry (SAM) and the group analysis of individual SAM data was performed using a permutation test.

Results: Our results revealed bilateral activation of the caudolateral primary somatosensory cortex following sensory pharyngeal stimulation with a slight lateralization to the side of stimulation.

Conclusion: The method introduced here is simple and easy to perform and might be applicable in the clinical setting. The results are in keeping with previous findings showing bihemispheric involvement in the complex task of sensory pharyngeal processing. They might also explain changes in deglutition after hemispheric strokes. The ipsilaterally lateralized processing is surprising and needs further investigation.
\end{abstract}

\section{Background}

Dysphagia is a common complication of a variety of neurological diseases affecting both the peripheral and central nervous system. Thus, dysphagia has been reported in neurologic patients suffering from ischemic stroke, amyotrophic lateral sclerosis, Kennedy disease, myasthenia gravis and Parkinson disease as well as ENT patients with tumors or due to postoperative lesions. The consequences of oropharyngeal dysphagia can be severe: dehydration, malnutrition, aspiration, choking, pneumonia, and death. Nursing home occupants with oropharyngeal dysphagia and aspiration have 45\% 12-month mortality [1].

Processing of oropharyngeal sensory information is crucial to assure safe deglutition. Impairments of sensation, as seen in stroke patients, causes severe swallowing prob- 
lems [2-6]. Even induced oropharyngeal anaesthesia is known to result in short-term dysphagia in healthy subjects [7-9].

During the last years, the interest in swallowing, dysphagia and oropharyngeal sensory processing has been constantly growing. Several studies have examined the cortical activation of human swallowing [10-20] finding bilateral processing in the primary somatosensory cortex during swallowing execution. Reduced sensory input, due to local oropharyngeal anesthesia, leads to significantly reduced bilateral cortical activation during swallowing processing in healthy subjects [21].

Little is known about cortical processing of sensory pharyngeal stimulation. In the present study we examined nine healthy subjects by means of magnetoencephalography (MEG) and synthetic aperture methods (SAM). In each subject both sides of the pharyngeal wall were stimulated. We hypothesized bilateral activation of the lateral sensorimotor cortex following sensory pharyngeal stimulation.

\section{Results}

All participants tolerated tube placement and air puff application without any difficulties. No coughing and especially no signs of aspiration occurred during tube placement and measurements. No swallowing was elicited by the pneumatic stimulation. None of the subjects complained about an urge to swallow due to the stimulation. Subjects were instructed to swallow between the air pulses. Therefore we tried to reduce movement artifacts in the examined time interval. Localization of the stimulation area was depending on the statement of the subjects. The position of the tube was adjusted until each subject stated sensory sensation at the lateral pharyngeal wall corresponding to the chosen nostril. During and after each MEG recording session subjects had to state whether the
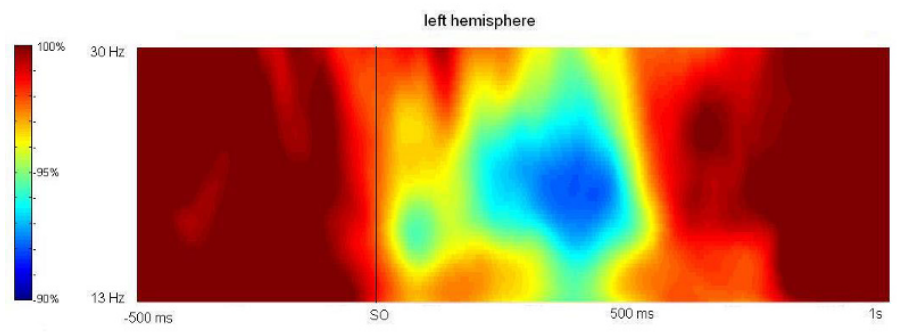

stimulation area was unchanged. If the tube position changed during recording, the whole measurement was repeated. This problem occurred only once.

Wavelet analysis of virtual channel recordings over the individual maximum event related desynchronization (ERD) in each hemisphere revealed a reduction of power in the beta frequency range directly after stimulus onset. A re-increase of power was found after ending of sensory stimulation. ANOVA and post-hoc t-tests revealed a decrease of beta power from the 'control time window' to the 'active time window' after stimulation to both sides of the pharyngeal wall and in both hemispheres [see figure 1].

According to these time-frequency plots SAM analysis was calculated for the two relevant frequency bands, alpha and beta comparing the 'active time window' to the 'control time window'.

Individual SAM analysis of the alpha and beta frequency band resulted in bilateral ERD within the caudolateral primary somatosensory cortex for both stimulation sides in all subjects.

SAM group analysis resulted in significant beta ERD for both stimulated sides $(\mathrm{p}<0.05)$. Again maximum ERD were located bilaterally within the caudolateral primary somatosensory cortex, corresponding to Brodmann areas (BA) 1, 2, and 3 but also spread into the motor cortex and secondary somatosensory areas (BA 4, 6, 5, and 40) [see table 1 and figure 2]. No significant activation in group analysis was observed in any other cortical area or in the alpha frequency range.

Regarding hemispheric lateralization stronger activation was observed in the ipsilateral hemisphere side. Stimulation of the left pharyngeal wall resulted in a left lateralized

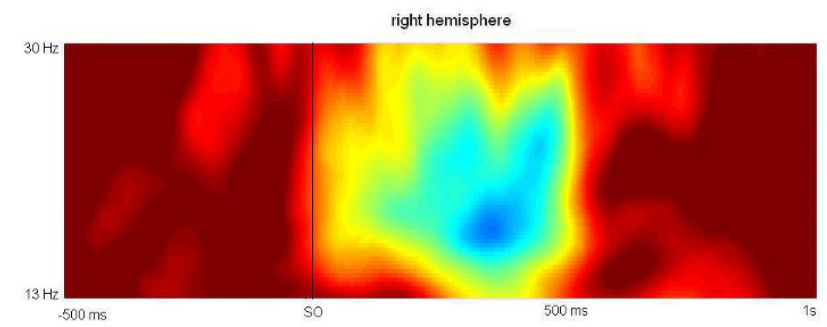

Figure I

Time-frequency wavelet plots. Wavelet analysis of the virtual channels representing the individual maximum ERD (left: maximum ERD over the left hemisphere; right: maximum ERD over the right hemisphere). Colors represent the percental change of frequency power relative to baseline (100\%) as indicated in the color bar. Changes relative to the baseline interval ($0.5-0 \mathrm{sec}$., whereas 0 is $\mathrm{SO}$ ) are calculated separately for each frequency. The time points used to define the time intervals of interest are marked ( $\mathrm{SO}=$ stimulus onset). A distinct decrease of frequency power in the beta frequency range after stimulus onset and a re-increase after pneumatic stimulation stopped is found. The effect is comparable in both hemispheres. 
a)
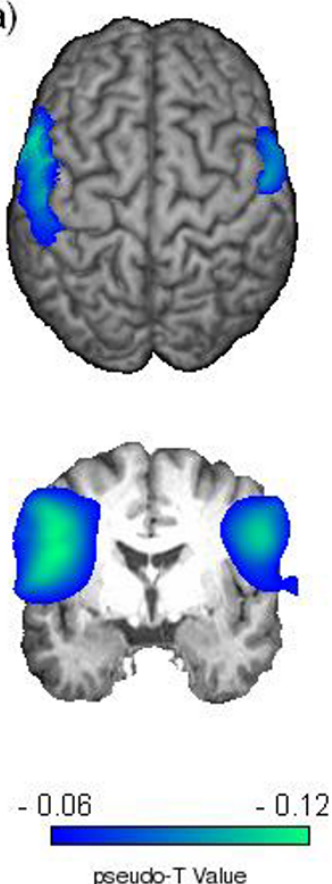

0.12

pseudo-T Value
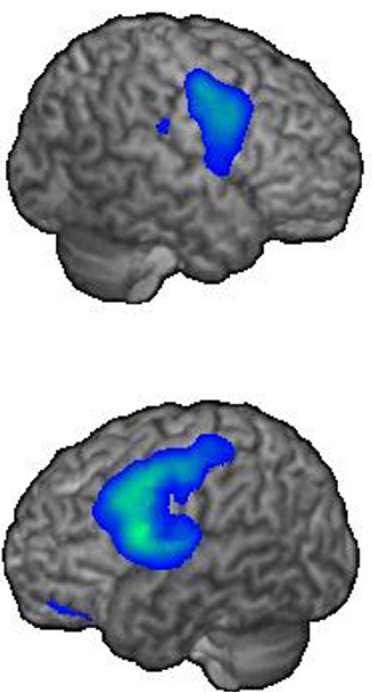

b)
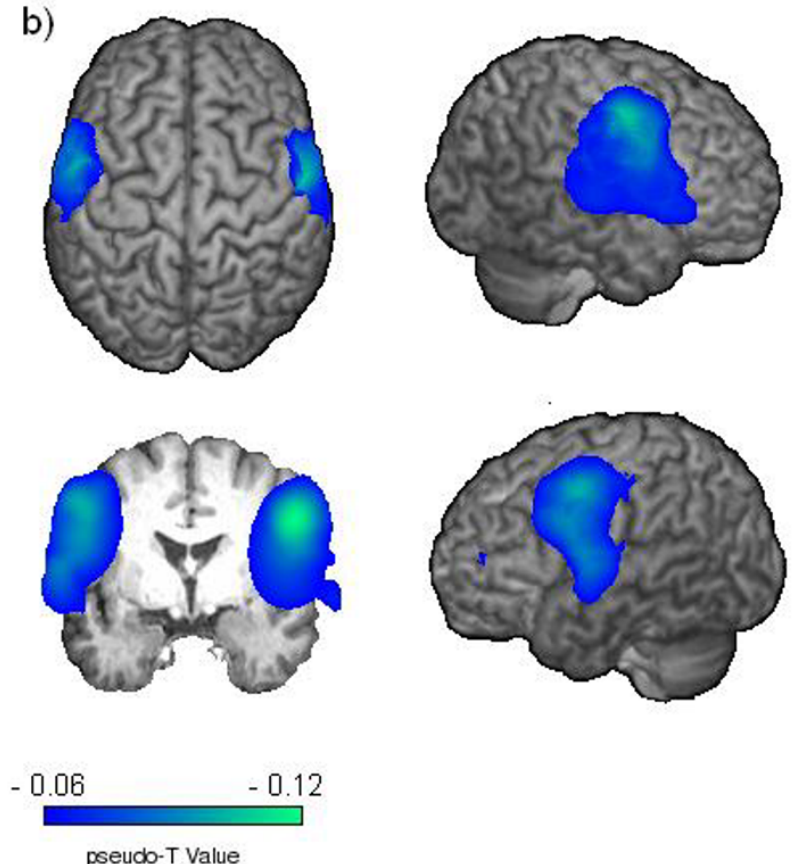

pseudo-T Value

Figure 2

Event related desynchronization. Significant activation in group analysis is shown $(p<0.05)$. Changes in the beta-frequency-band during pneumatic pharyngeal activation compared to the resting stage. a) ERD evoked by pneumatic stimulation to the left side of the pharyngeal wall. Stronger and broader activation is found in the temporal lobe of the left hemisphere. b) ERD evoked by pneumatic stimulation of the right side of the pharyngeal wall. Here a right hemispheric lateralization of temporal activation can be seen. The color bar represents the t-value.

cortical activation (LI: 0.61), while a reverse, but much weaker effect was seen in right sided stimulation (LI: $0.16)$.

\section{Discussion}

This is one of the first studies presenting a simple and easy to perform paradigm to examine the cortical processing of sensory pharyngeal stimulation by means of MEG. The pneumatic stimulation of the pharyngeal wall was well tolerated by all subjects and led to bilateral activation of the caudolateral primary somatosensory cortex. The exact stimulation location cannot be detected. Therefore we were only depending on the statement of the subjects. A bilateral stimulation due to malpositioning or tube movement cannot definitely be excluded.

Some previous MEG and EEG studies found changes in the central alpha rhythm linked to the somatosensory system, while beta changes are linked to the motor system [22]. In contrast to this significant activation was only found in the beta frequency range in the present study.

Table I: Talairach coordinates and the corresponding Brodmann area of task-related activation across the two different stimulation sides in both hemispheres are shown.

\begin{tabular}{ccccc}
\hline Stimulation & Right & Right & Left & Left \\
\hline Hemisphere & Right & Left & Right & Right \\
\hline Talairach Coordinate & $62.37,-10.43,24.47$ & $-57.42,-14.91,32.07$ & $63.36,-9.55,22.59$ & $-54.45,-10.43,43.82$ \\
\hline Brodmann Area & 3 & 3 & 3 & 3 \\
\hline
\end{tabular}


The activated somatosensory cortex is part of the facial area and known to process afferent pharyngeal information since Brodmann [23]. In a MEG study electrical stimulation of the pharynx elicited activation in the same area of the primary somatosensory cortex [24]. The localization has also been confirmed by positron emission tomography [12] and in epilepsy surgery [25]. Also two recent fMRI studies examining pneumatic stimulation of the oropharynx found bilateral activation of the sensorimotor cortex [26,27].

While the relevance of sensory information for secure deglutition is beyond controversy, a specific examination of its cortical processing has rarely been performed. An increased cortical excitability after electrical pharyngeal stimulation and a somatotopic cortical representation of swallowing related musculature could be shown by transcranial magnetic stimulation [28]. Up to now, examinations of the neuronal activation after pharyngeal stimulation mainly addressed therapeutic options for dysphagic patients. Transcranial magnetic stimulation studies demonstrated that peripheral intraluminal low-frequency stimulation in humans resulted in increased corticobulbar excitability in health and after stroke [29,30]. Other studies focused on the influence of pharyngeal stimulation on deglutition. Cold and taste stimulation of the faucial area hastens the onset of the pharyngeal swallowing phase and reduces swallowing latency $[31,32]$. Electrical stimulation of the superior laryngeal nerve in cats resulted in reduced swallowing latency [33].

The second and more surprising finding of this study was a slight ipsilateral lateralized cortical processing following pharyngeal stimulation. Afferent information of the oropharynx is transferred by the glossopharngeal nerve to the nucleus spinalis nervi trigemini located in the brain stem. After crossing the midline it ascends to the thalamus and afterwards reaches the primary somatosensory cortex $[34,35]$. Decussation of sensory as well as motor fibres is one of the main principles of the central nervous system. Altogether about $90 \%$ of these fibres cross over to the contralateral side in the mesencephalon. This leads to a strong contralateral lateralization of most cerebral processes which have been demonstrated frequently for the auditory, visual, sensory and motor system. Only for the olfactory system a mainly ipsilateral cortical processing has been shown [36]. Therefore the bilateral and even slight ipsilateral lateralized processing found in our MEG data is quite remarkable. A similar effect has previously been shown after repetitive transcranial magnetic stimulation of the pharyngeal motor cortex. Here a visible stronger and longer ipsilateral activation was seen. Nevertheless this observation did not reach significance during further calculations [37]. In an fMRI study oral stimulation of only the right side resulted in bilateral cortical activation
[27]. Our results are also supported by previous findings showing bilateral hemispheric involvement in the complex task of sensory pharyngeal processing in a PET study [12]. Bihemispheric involvement is also reflected in patients with hemispheric stroke. Both right and left hemispheric strokes can lead to sensory impairment of the oropharyngeal area resulting in severe deglutition $[2,38,39]$. It also correlates with a bilateral motor activation seen in reflexive swallowing [10]. A recent fMRI study found bilateral sensorimotor activation with a slightly contralateral lateralization after oropharyngeal stimulation [26]. Apart from methodological differences the stimulated areas varied from those in our study. Sörös et al. focused on the oral part of the pharyngeal cavity and therefore stimulation was delivered through a dental splint. Contrary to this the stimulation in our study was performed using a nasal tube and delivered to the lower part of the pharynx. The difference of stimulation locality might explain the reverse lateralization in cortical activation.

We therefore conclude that the anatomical decussation of the pharyngeal afferences does not lead to a functional correlate. The ipsilateral hemisphere seems to be at least as involved in sensory oropharyngeal input as the contralateral one.

The pneumatic stimulation paradigm is able to indicate the corresponding cortical areas processing pharyngeal sensor information. This easy and quite non invasive measurement is objective and can also be performed on different groups of dysphagic patients to increase our understanding about the physiology and pathophysiology of pharyngeal sensor processing. It might also be helpful to monitor swallowing recovery after stroke as well as other neurogenic or morphologic alterations leading to dysphagia or even to observe and compare different therapies.

\section{Conclusion}

In conclusion the new stimulation method introduced here is in principle simple and easy to perform and might therefore also be applicable in the clinical setting. The results reveal bilateral hemispheric involvement in the complex task of sensory pharyngeal processing. They might also explain frequently changes in deglutition after hemispheric strokes, showing likewise disturbances after right as well as left hemispheric stroke. Additionally, the slight ipsilaterally lateralized processing is surprising and needs further investigation.

\section{Methods \\ Subjects}

Nine healthy right-handed volunteers ( 5 males, age range 23 - 35 years, mean 29 years) served as subjects. The local 
regional ethics committee approved the protocol of the study. Informed consent was obtained from each subject after the nature of the study was explained in accordance to the principles of the Declaration of Helsinki.

\section{Sensory pharyngeal stimulation}

A baby gastric tube (Unomedical, Sterile EO, $40 \mathrm{~cm}$ length, $11 / 3 \mathrm{~mm}$ diameter) was used for pneumatic stimulation. Air puffs were administered by compressed oxygen with a flow of $2 \mathrm{l} / \mathrm{min}$ through the tube. One of the two lateral apertures of the gastric tube was sealed and the tube was placed through one nostril and advanced forward until visible under the velum. The position of the tube tip was adjusted until the subject stated to feel the air puffs laterally on the pharyngeal wall at the side of the nostril the tube was placed in [see figure 3].

A 24 minute measurement was performed with the air puffs being applied with a stimulation frequency of 0.5 $\mathrm{Hz}$ and $500 \mathrm{~ms}$ stimulus duration. Stimulus duration was chosen to gain a reasonable long time interval for SAM analyses. The inter-stimulus interval in MEG studies with somatosensory stimulation is varying between 0.33 and 3 seconds in the actual literature [40-44]. In the present study the stimulus frequency of $0.5 \mathrm{~Hz}$ was chosen to keep the measurement duration short on the one hand and gaining sufficient recovery of the active nerve cells on the

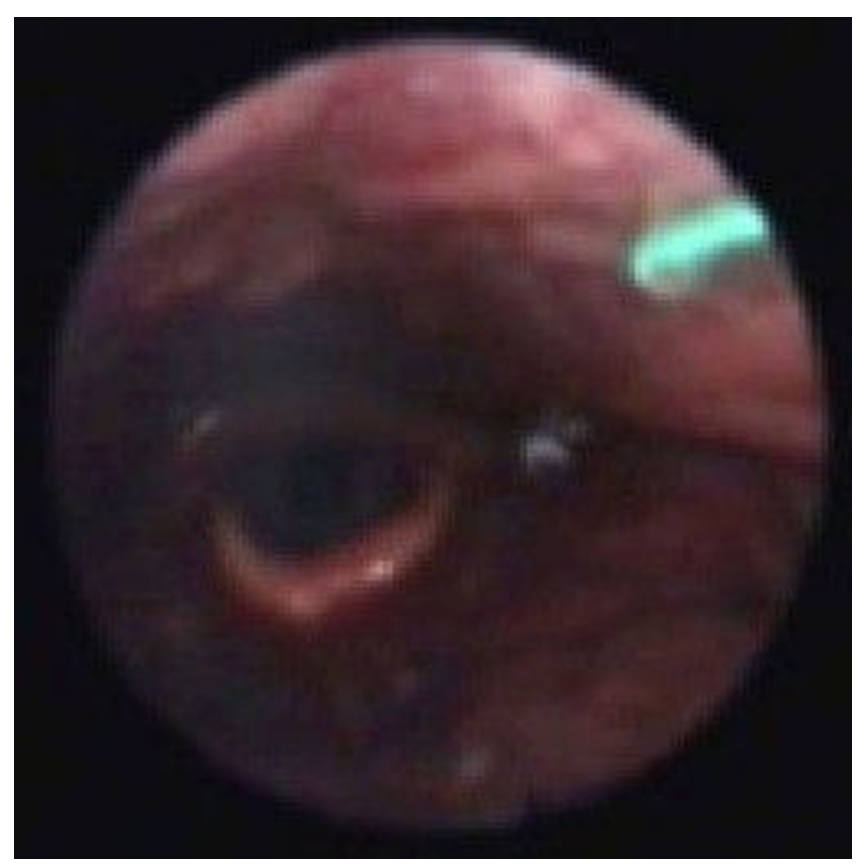

\section{Figure 3}

Position of the nasogastric tube. The endoscopic picture illustrates the position of the gastric tube during pneumatic stimulation in one of the subjects. In this picture the tip of the tube is placed through the left nostril. other hand. A 5 liter bottle of oxygen was connected to a pneumatic stimulation device. During stimulation an oxygen flow of $2 \mathrm{l} / \mathrm{min}$ was chosen.

\section{MEG recording}

A whole head 275-channel SQUID sensor array (Omega 275, CTF Systems Inc.) was used to collect MEG data with a sample frequency of $600 \mathrm{~Hz}$. Data was filtered during acquisition using a $150 \mathrm{~Hz}$ low-pass filter. Subjects were seated in a comfortably upright position watching a self selected silent movie.

\section{Anatomical MRI}

MRI data were acquired on a 3.0 T Scanner (Gyroscan Intera, Philips) with a standard head coil. T1-weighted sagittal anatomical images with in-plane resolution of $512 \times 512(0.6 \times 0.6 \mathrm{~mm}$ resolution $)$ and 320 slices $(0.5$ $\mathrm{mm}$ thickness) were recorded using spoiled gradient echo imaging.

\section{Data analysis}

To define the active frequency bands time-frequency plots were calculated using wavelet analysis. These calculations were done using EMEGS (ElectroMagnetic-EncephaloGraphy Software; http://www.emegs.org), a tool for analyzing neuroscientific data developed in MATLAB [45]. The 275 channels of the MEG system were fragmented into 10 channel groups, frontal, central, parietal, temporal and occipital channels in each hemisphere. Data from each individual subject was averaged across trials (-0.8 to $1.2 \mathrm{~s}$ in reference to stimulus onset) and time-frequency analysis was performed $(0-150 \mathrm{~Hz})$. The time-frequency plots of the parietal channels were determined for both hemispheres and averaged across all subjects in each group.

According to the changes of the time-frequency analysis MEG data were than filtered within two frequency bands: alpha $(8-13 \mathrm{~Hz})$ and beta $(13-30 \mathrm{~Hz})$. SAM was used to generate a $20 \times 20 \times 14 \mathrm{~cm}^{3}$ volumetric pseudo-t images [46] from the filtered MEG signals, with 3-mm voxel resolution. A pseudo-t value cancels the common-mode brain activity by subtracting the source power found in a defined control stage from the source power in the active stage. To account for uncorrelated sensor noise, this difference is normalized by the mapped noise power $[46,47]$. For analyzing cortical activity during "active time window" $(0-0.5)$ the corresponding "control time window" $(-0.5-0)$ served as control.

Group analysis of multiple subjects' data was performed as previously published $[22,48-50]$. Briefly, the individual MRIs were first transformed into a common anatomical space using SPM2. Then the spatial normalized activation maps were obtained by applying this transformation to the individual SAM volumes. The significance of activated 
brain regions was investigated by the permutation test for the observed time interval. To calculate difference between stimulation sides a standard permutation test for paired samples was performed [51].

Hemispheric lateralization of brain activation was quantified using a lateralization index (LI), which was calculated as $(\mathrm{L}-\mathrm{R}) /(\mathrm{L}+\mathrm{R})$, where $\mathrm{L}$ and $\mathrm{R}$ are the cumulative pseudo$\mathrm{t}$ activation in the somatosensory cortex (BA 3, 1 and 2, according to the Talairach atlas) of the left and right hemisphere, respectively. A positive LI indicates left hemispheric lateralization, while a negative LI indicates stronger right hemispheric activation. Ratios around 0 represent indeterminate dominance, 1 , respectively -1 are indicating unilateral activation $[10,52]$.

To examine the temporal sequencing of activation virtual channels were calculated individually for the maximum beta ERD in each hemisphere. Afterwards time-frequency plots of virtual channel activation were calculated and grand averaged using EMEGS. Afterwards the two time intervals "active time window" $(-0.5-0)$ and "control time window" $(0-0.5)$ were defined for further calculations. Comparisons between different stimulation sides, time intervals and hemispheres were performed using two-way ANOVA followed by post-hoc t-tests.

\section{Authors' contributions}

IT performed analysis and interpretation of data and drafted the manuscript. She was funded by the Deutsche Forschungsgemeinschaft. OS has made analysis and interpretation of data and was involved in drafting the manuscript. TW helped in the development of the paradigm and performed several of the measurements. CP and EBR revised the manuscript critically for important intellectual content. RD made substantial contributions to conception and design, and has given final approval of the version to be published. All authors read and approved the final manuscript

\section{Acknowledgements}

This study was supported by Deutsche Forschungsgemeinschaft (PA-392/92) and (JU 445/5-I).

\section{References}

I. Cook IJ, Kahrilas PJ: AGA technical review on management of oropharyngeal dysphagia. Gastroenterology 1999, I I 6(2):455-478

2. Aviv JE, Martin JH, Sacco RL, Zagar D, Diamond B, Keen MS, Blitzer $A$ : Supraglottic and pharyngeal sensory abnormalities in stroke patients with dysphagia. Ann Otol Rhinol Laryngol 1996, 105(2):92-97.

3. Daniels SK, Corey DM, Fraychinaud A, DePolo A, Foundas AL: Swallowing lateralization: the effects of modified dual-task interference. Dysphagia 2006, 2 I (I):2I-27.

4. Daniels SK, Brailey K, Priestly DH, Herrington LR, Weisberg LA, Foundas AL: Aspiration in patients with acute stroke. Arch Phys Med Rehabil 1998, 79(1):14-19.

5. Johnson ER, McKenzie SW, Sievers A: Aspiration pneumonia in stroke. Arch Phys Med Rehabil 1993, 74(9):973-976.
6. Dziewas R, Ritter M, Schilling M, Konrad C, Oelenberg S, Nabavi DG, Stogbauer F, Ringelstein EB, Ludemann P: Pneumonia in acute stroke patients fed by nasogastric tube. J Neurol Neurosurg Psychiatry 2004, 75(6):852-856.

7. Ertekin C, Kiylioglu N, Tarlaci S, Keskin A, Aydogdu I: Effect of mucosal anaesthesia on oropharyngeal swallowing. Neurogastroenterol Motil 2000, I 2(6):567-572.

8. Mansson I, Sandberg N: Manometry of the pharynx and the esophagus in relation to laryngectomy. JFORL J Fr Otorhinolaryngol Audiophonol Chir Maxillofac 1974, 23(8):737-743.

9. Teismann IK, Steinstraeter O, Stoeckigt K, Suntrup S, Wollbrink A, Pantev C, Dziewas R: Functional oropharyngeal sensory disruption interferes with the cortical control of swallowing. $B M C$ Neurosci 2007, 8(I):62.

10. Dziewas R, Soros P, Ishii R, Chau W, Henningsen H, Ringelstein EB, Knecht S, Pantev C: Neuroimaging evidence for cortical involvement in the preparation and in the act of swallowing. Neuroimage 2003, 20(I): |35-| 44.

II. Furlong PL, Hobson AR, Aziz Q, Barnes GR, Singh KD, Hillebrand A, Thompson DG, Hamdy S: Dissociating the spatio-temporal characteristics of cortical neuronal activity associated with human volitional swallowing in the healthy adult brain. Neuroimage 2004, 22(4): | 447-I 455.

12. Hamdy S, Rothwell JC, Brooks DJ, Bailey D, Aziz Q, Thompson DG: Identification of the cerebral loci processing human swallowing with H2(I5)O PET activation. I Neurophysiol 1999, $81(4): 1917-1926$

13. Hamdy S, Mikulis DJ, Crawley A, Xue S, Lau H, Henry S, Diamant NE: Cortical activation during human volitional swallowing: an event-related fMRI study. Am J Physiol 1999, 277(I Pt I):G219-225.

14. Kern MK, Jaradeh S, Arndorfer RC, Shaker R: Cerebral cortical representation of reflexive and volitional swallowing in humans. $\quad A m$ I Physiol Gastrointest Liver Physiol 200I, 280(3):G354-360.

15. Martin RE, Maclntosh BJ, Smith RC, Barr AM, Stevens TK, Gati JS, Menon RS: Cerebral areas processing swallowing and tongue movement are overlapping but distinct: a functional magnetic resonance imaging study. I Neurophysiol 2004, 92(4):2428-2443

16. Mosier KM, Liu WC, Maldjian JA, Shah R, Modi B: Lateralization of cortical function in swallowing: a functional MR imaging study. AJNR Am J Neuroradiol 1999, 20(8): I520-1526.

17. Hamdy S, Rothwell JC, Aziz Q, Singh KD, Thompson DG: Longterm reorganization of human motor cortex driven by short-term sensory stimulation. Nat Neurosci 1998, I(I):64-68.

18. Huckabee ML, Deecke L, Cannito MP, Gould HJ, Mayr W: Cortical control mechanisms in volitional swallowing: the Bereitschaftspotential. Brain Topogr 2003, 16(1):3-17.

19. Dziewas R, Soros P, Ishii R, Chau W, Henningsen H, Ringelstein EB, Knecht S, Pantev C: Cortical processing of esophageal sensation is related to the representation of swallowing. Neuroreport 2005, 16(5):439-443.

20. Teismann IK, Dziewas R, Steinstraeter O, Pantev C: Time-dependent hemispheric shift of the cortical control of volitional swallowing. Hum Brain Mapp 2009, 30(4): 1352-60.

21. Teismann IK, Steinstraeter O, Stoeckigt K, Suntrup S, Wollbrink A, Pantev C, Dziewas R: Functional oropharyngeal sensory disruption interferes with the cortical control of swallowing. $B M C$ Neurosci 2007, 8:62.

22. Taniguchi M, Kato A, Fujita N, Hirata M, Tanaka H, Kihara T, Ninomiya H, Hirabuki N, Nakamura H, Robinson SE, et al.: Movement-related desynchronization of the cerebral cortex studied with spatially filtered magnetoencephalography. Neuroimage 2000, 12(3):298-306.

23. Brodmann K: Special functional localisation. In Brodmann's Localisation in the Cerebral Cortex: The Principles of Comparative Localasation in the Cerebral Cortex Based on Cytoarchitectonics 3rd edition. Springer Verlag; 2005:254-63.

24. Gow D, Hobson AR, Furlong P, Hamdy S: Characterising the central mechanisms of sensory modulation in human swallowing motor cortex. Clin Neurophysiol 2004, I I 5( I 0):2382-2390.

25. Lehman R, Andermann F, Olivier A, Tandon PN, Quesney LF, Rasmussen TB: Seizures with onset in the sensorimotor face area: clinical patterns and results of surgical treatment in $\mathbf{2 0}$ patients. Epilepsia 1994, 35(6): I I I7-I I 24. 
26. Soros P, Lalone E, Smith R, Stevens T, Theurer J, Menon RS, Martin RE: Functional MRI of oropharyngeal air-pulse stimulation. Neuroscience 2008, I 53(4): I300- I308.

27. Lowell SY, Poletto CJ, Knorr-Chung BR, Reynolds RC, Simonyan K Ludlow CL: Sensory stimulation activates both motor and sensory components of the swallowing system. Neuroimage 2008, 42(I):285-295.

28. Hamdy S, Aziz Q, Rothwell JC, Singh KD, Barlow J, Hughes DG, Tallis RC, Thompson DG: The cortical topography of human swallowing musculature in health and disease. Nat Med 1996 2(I I): $1217-1224$.

29. Fraser C, Power M, Hamdy S, Rothwell J, Hobday D, Hollander I Tyrell P, Hobson A, Williams S, Thompson D: Driving plasticity in human adult motor cortex is associated with improved motor function after brain injury. Neuron 2002, 34(5):83 I-840.

30. Power M, Fraser C, Hobson A, Rothwell JC, Mistry S, Nicholson DA, Thompson DG, Hamdy S: Changes in pharyngeal corticobulbar excitability and swallowing behavior after oral stimulation. Am J Physiol Gastrointest Liver Physiol 2004, 286(I):G45-50.

31. Kaatzke-McDonald MN, Post E, Davis PJ: The effects of cold, touch, and chemical stimulation of the anterior faucial pillar on human swallowing. Dysphagia 1996, I I(3): I98-206.

32. Lazzara L, Lazarus C, Logemann J: Effects of thermal stimulation on patients with swallowing disorders - A videofluoroscopic analysis. Dysphagia 1986, I:73-33.

33. Chi-Fishman G, Capra NF, McCall GN: Thermomechanical facilitation of swallowing evoked by electrical nerve stimulation in cats. Dysphagia 1994, 9(3): |49-155.

34. Mu L, Sanders I: Sensory nerve supply of the human oro- and laryngopharynx: a preliminary study. Anat Rec 2000, 258(4):406-420.

35. Yoshida $Y$, Tanaka $Y$, Hirano $M$, Nakashima T: Sensory innervation of the pharynx and larynx. Am J Med 2000, I08(Suppl 4a):5IS-6IS

36. Tonoike M, Yamaguchi M, Kaetsu I, Kida H, Seo R, Koizuka I: Ipsilateral dominance of human olfactory activated centers estimated from event-related magnetic fields measured by $\mathbf{I} 22$ channel whole-head neuromagnetometer using odorant stimuli synchronized with respirations. Ann N Y Acad Sci 1998 , 855:579-590.

37. Gow D, Rothwell J, Hobson A, Thompson D, Hamdy S: Induction of long-term plasticity in human swallowing motor cortex following repetitive cortical stimulation. Clin Neurophysiol 2004, I I 5(5): I044-105I.

38. Ding R, Logemann JA: Pneumonia in stroke patients: a retrospective study. Dysphagia 2000, I 5(2):5I-57.

39. Mann G, Hankey GJ, Cameron D: Swallowing function after stroke: prognosis and prognostic factors at $\mathbf{6}$ months. Stroke 1999, 30(4):744-748.

40. Hoechstetter K, Rupp A, Stancak A, Meinck HM, Stippich C, Berg P, Scherg $M$ : Interaction of tactile input in the human primary and secondary somatosensory cortex - a magnetoencephalographic study. Neuroimage 200I, I4(3):759-767.

4I. Zhu Z, Disbrow EA, Zumer JM, McGonigle DJ, Nagarajan SS: Spatiotemporal integration of tactile information in human somatosensory cortex. BMC Neurosci 2007, 8:21.

42. Wegner K, Forss N, Salenius S: Characteristics of the human contra-versus ipsilateral SII cortex. Clin Neurophysiol 2000 I I I(5):894-900.

43. Forss N, Salmelin R, Hari R: Comparison of somatosensory evoked fields to airpuff and electric stimuli. Electroencephalogr Clin Neurophysiol 1994, 92(6):510-517.

44. Karageorgiou E, Koutlas IG, Alonso AA, Leuthold AC, Lewis SM, Georgopoulos AP: Cortical processing of tactile stimuli applied in quick succession across the fingertips: temporal evolution of dipole sources revealed by magnetoencephalography. Exp Brain Res 2008, I 89(3):3||-32|.

45. Junghoefer M, Peyk P: Analysis of electrical potentials and magnetic fields of the brain. Matlab Select 2004:24-28.

46. Vrba J, Robinson SE: Signal processing in magnetoencephalography. Methods 200I, 25(2):249-27I.

47. Sekihara K, Sahani M, Nagarajan SS: Localization bias and spatial resolution of adaptive and non-adaptive spatial filters for MEG source reconstruction. Neuroimage 2005, 25(4): $1056-1067$
48. Chau W, Herdman AT, Picton TW: Detection of power changes between conditions using split-half resampling of synthetic aperture magnetometry data. Neurol Clin Neurophysiol 2004, 2004:24.

49. Hirata M, Kato A, Taniguchi M, Ninomiya H, Cheyne D, Robinson SE, Maruno M, Kumura E, Ishii R, Hirabuki N, et al.: Frequencydependent spatial distribution of human somatosensory evoked neuromagnetic fields. Neurosci Lett 2002, 3 I 8(2):73-76.

50. Chau W, Mclntosh AR, Robinson SE, Schulz M, Pantev C: Improving permutation test power for group analysis of spatially filtered MEG data. Neuroimage 2004, 23(3):983-996.

5I. Nichols TE, Holmes AP: Nonparametric permutation tests for functional neuroimaging: a primer with examples. Hum Brain Mapp 2002, I 5(I): I-25.

52. Yetkin FZ, Hammeke TA, Swanson SJ, Morris GL, Mueller WM, McAuliffe TL, Haughton VM: A comparison of functional MR activation patterns during silent and audible language tasks. AJNR Am J Neuroradiol 1995, I 6(5): 1087-1092.
Publish with Biomed Central and every scientist can read your work free of charge

"BioMed Central will be the most significant development for disseminating the results of biomedical research in our lifetime. "

Sir Paul Nurse, Cancer Research UK

Your research papers will be:

- available free of charge to the entire biomedical community

- peer reviewed and published immediately upon acceptance

- cited in PubMed and archived on PubMed Central

- yours - you keep the copyright
BiolMedcentral 